\title{
Expression of HMG1 and metastasis of colorectal cancer
}

\author{
Viroj Wiwanitkit
}

Accepted: 27 October 2009/Published online: 18 November 2009

(C) Springer-Verlag 2009

\section{Dear Editor:}

I read the recent publication by Huang et al. with a great interest. The expression on high-mobility group box-1 (HMG1) and its correlation to metastasis in colorectal cancer is reported in this study. Huang et al. reached to the conclusion that "The HMGI-C and HMGI(Y) quantitations by real-time RT-PCR are associated with Dukes' staging and metastasis; hence, the gene expression levels may be useful in clinical practice." Of interest, the role of HMG1 has been mentioned for a few years. Sasahira et al. (2005) proposed for the malignant nature of the tumor in case with expression of HMG1. Kuniyasu et al. (2003) reported that the coexpression of HMG1 and receptor for advanced glycation end products (RAGE) was closely associated with invasion and metastasis of colorectal cancer. RAGE seems to be a big confounding parameter in the study of HMG1 and progression of the cancer. Further analysis on coexpression of RAGE in the study of Huang et al. might add up the value of the study. 